GA-A24521

\title{
THE LOCAL LIMIT OF GLOBAL GYROKINETIC SIMULATIONS
}

by
J. CANDY, R.E. WALTZ, and W. DORLAND 


\section{DISCLAIMER}

This report was prepared as an account of work sponsored by an agency of the United States Government. Neither the United States Government nor any agency thereof, nor any of their employees, makes any warranty, express or implied, or assumes any legal liability or responsibility for the accuracy, completeness, or usefulness of any information, apparatus, product, or process disclosed, or represents that its use would not infringe privately owned rights. Reference herein to any specific commercial product, process, or service by trade name, trademark, manufacturer, or otherwise, does not necessarily constitute or imply its endorsement, recommendation, or favoring by the United States Government or any agency thereof. The views and opinions of authors expressed herein do not necessarily state or reflect those of the United States Government or any agency thereof. 
GA-A24521

\title{
THE LOCAL LIMIT OF GLOBAL GYROKINETIC SIMULATIONS
}

\author{
by
J. CANDY, R.E. WALTZ, and W. DORLAND*
}

This is a preprint of a paper to be submitted for publication in Phys. Plasmas.

*University of Maryland, College Park, Maryland.

\author{
Work supported by \\ the U.S. Department of Energy under \\ Grant Nos. DE-FG03-95ER54309 and DE-FG02-93ER54197
}

GENERAL ATOMICS PROJECT 03726

OCTOBER 2003 


\begin{abstract}
Global gyrokinetic simulations of turbulence include physical effects that are not retained in local flux-tube simulations. Nevertheless, in the limit of sufficiently small $\rho_{*}$ (gyroradius compared to system size) it is expected that a local simulation should agree with a global one (at the local simulation radius) since all effects that are dropped in the local simulations are expected to vanish as $\rho_{*} \rightarrow 0$. In this note, global simulations of a well-established test case are indeed shown to recover the flux-tube limit at each radius.
\end{abstract}


The purpose of this letter is to clarify the connection between local and global gyrokinetic (GK) turbulence simulations. In previous work [1] using the GYRO code [2], a study of the mechanisms by which transport scaling can be degraded from gyroBohm to Bohm, or to worse than Bohm, was made for ion temperature gradient (ITG) turbulence with adiabatic electrons. The critical role played by equilibrium profile shearing, particularly in cases near marginal stability, was discussed. A recurring theme in Ref. [1] was that the value of $\chi_{i}$ observed in a global simulation with sufficiently small value of $\rho_{*}$ is the same as that found in the corresponding local (flux-tube) simulation. Here, $\rho_{*} \doteq \rho_{s} / a$ with $\rho_{s} \doteq c_{s} / \Omega_{c i}$ the ion-sound gyroradius, $c_{s}=\sqrt{T_{e} / m_{i}}$ the sound speed, and $\Omega_{c i}$ the ion gyrofrequency.

At about the same time, Lin, et al. [3] reported work on the size scaling of turbulent transport using a different code (GTC) and with a specific set of equilibrium profiles. Unlike GYRO, which uses an Eulerian discretization scheme, the GTC code uses the popular particle-in-cell (PIC) method to solve the GK equations. A discussion of the relative merits of each scheme is beyond the scope of the present paper and can be found elsewhere $[4,5]$. Suffice it to say that because the two codes use radically different numerical methods, they are susceptible to radically different types of discretization error. In the study reported in Ref. [3], adiabatic electron dynamics and finite-aspect-ratio toroidal geometry were considered. A parameter scan was given for $1 / \rho_{*}$ in the range $125 \leq 1 / \rho_{*} \leq 1000$. The overarching result was to show that "the local transport coefficient exhibits a gradual transition from a Bohm-like scaling for devices sizes corresponding to present-day tokamak experiments to a gyroBohm scaling for future larger devices." We remark that the scenario studied in Ref. [3] was constructed so as to match the DIII-D Cyclone Base Case at $r / a=0.5$ [6]. However, the results in Ref. [3] showed an obvious anomaly: at $1 / \rho_{*}=1000$, the asymptotic value of the transport coefficient was $\chi_{i} \simeq 3.4 \chi_{\mathrm{GB}}$, about $80 \%$ larger than the well-established local value of Dimits: $\chi_{i} \simeq 1.9 \chi_{\mathrm{GB}}$ (see LLNL GK result in Ref. [6]). Here, $\chi_{\mathrm{GB}} \doteq \rho_{s}^{2} c_{s} / a$ defines the gyroBohm normalization. Note that Ref. [6] used a different normalization, $\chi_{\text {Dimits }} \doteq \rho_{s}^{2} c_{s} / L_{n}$, such that $\chi_{\mathrm{GB}}=1.25 \chi_{\text {Dimits. }}$. Subsequent investigations by Lin [7] showed that part of the discrepancy was due to a difference in the plasma equilibrium model used in Ref. [3], such that new simulations using "simplified geometry" (we believe that this means $s-\alpha$, but no details are given) gave an asymptotic result at least as large as $\chi_{i} \simeq 2.6 \chi_{\mathrm{GB}}$. This result is closer to, but still $35 \%$ higher than, the accepted value. The Dimits value, which is in close agreement with the GYRO Eulerian calculation, was obtained using a flux-tube GK-PIC code. This implies that the source of the GTC discrepancy cannot be based solely on choice of algorithm.

In an effort to understand these results, we made an exhaustive study of the GTC $\rho_{*}$-scan using GYRO. A variety of resolution tests and sensitivity studies were carried out in an effort to find some effect which would increase the transport 
to levels consistent with Ref. [7]. In the end, our results repeatedly showed what we expected all along: that global simulation results approach the flux-tube limit (with corresponding gyroBohm scaling), and do so at a system size characteristic of medium-size tokamaks (DIII-D L-mode plasmas have $1 / \rho_{*} \simeq 300$ ). As an added consistency check, we have also verified that the small- $\rho_{*}$ GYRO results agree with a series of simulations from the flux-tube Eulerian code GS2 [8], as will be shown later.

The generally unstated assumption in most simulation papers is that quoted values of $\chi_{i}$ have reached a statistical steady-state, and are adequately converged with respect to time-step and grid resolution. It is quite impractical to prove convergence for every case by running highly over-resolved simulations. In practice this is only done for a few cases, and judgments based on past experience are made for most cases. All GYRO cases reported in this paper used 128 points in velocity space ( 8 pitch angles, 8 energies and 2 signs of velocity), and radial grid spacing $\Delta r=\rho_{s}$. An adaptive long-wavelength source is also used to prevent profile relaxation, as detailed in Ref. [1]. Because of the spectral decomposition in the toroidal direction, GYRO can efficiently resolve cases at arbitrarily small $\rho_{*}$ by choosing $\Delta n>1$ (thereby simulating a toroidal wedge of width $2 \pi / \Delta n$ ). Here, $n$ is the toroidal mode number. In this letter, we used between 16 and 64 toroidal modes, as needed, although our experience is that rarely are cases encountered for which more than 32 modes are required.

With regard to the time domain, it is problematic in practice to determine whether or not a very expensive case is really in a stationary state having no trend, simply because it costs twice as much to run twice as long. In the end, there is no practical way to avoid the need for "good judgment". A key advantage of Eulerian codes, however, is the absence of noise build-up with run time, so that the existence of a stationary state can in principle be demonstrated. Inexpensive GYRO cases have been run to time scales ten times longer than those shown here with no indication of up- or down-trends in $\chi_{i}$. Furthermore, in GYRO, we have verified the neutral linear stability of the $n=0$ radial modes (zonal flows) for very long times. This is an important and strenuous test for cases with large radial domain size.

We summarize the present state of comparison between GYRO and published GTC results in Fig. 1, which shows the gyroBohm-normalized ion thermal diffusivity, $\chi_{i} / \chi_{\mathrm{GB}}$ versus $1 / \rho_{*}$ for each code. In Ref. [7] it is argued that the difference between the gyroBohm limit (horizontal line) and the GTC value is a consequence of nonperiodic boundary conditions, large radial domain size and radial variation of $\omega_{*}, q, s$ and $r / R$ used in the latter calculation. However, our results show that this explanation is not valid, since GYRO includes all these aforementioned effects yet agrees with the local result. There is also the unexplored possibility that some 
artifact of the GTC source, which acts to maintain the equilibrium profiles, gives rise to the difference.

While the nonspecialist would probably assume that global gyrokinetic codes would readily accept as input arbitrary temperature and density profiles, this is not generally the case. There are certain assumptions of nonphysical origin which are routinely made in global codes in order to facilitate simulations. One of the more common and problematic of these is the radial shape of the inverse-temperaturegradient scale-length profile, $1 / L_{T}(r) \doteq-(1 / T)(d T / d r)$. The customary setup $[3,9-13]$ for a global GK-PIC simulation is to place the largest instability drive at the center of the simulation domain, with vanishing drive near the simulation boundaries. In typical L-mode and H-mode discharges, however, $1 / L_{T}$ does not show this trend but rather tends to increase from core to edge, rising sharply near the edge. This is shown in Fig. 2 for a DIII-D L-mode discharge (\# 101381) we have simulated elsewhere [14] using full-physics and actual experimental profiles in real shaped geometry. Flux-tube codes, on the contrary, can only treat flat profiles across the radial simulation domain (in practice, a flux-tube simulation describes transport over a small fraction of the minor radius). One obvious feature of the GK-PIC gradient profiles shown in Fig. 2 is the central peaking that is present neither in experiments nor in flux-tube simulations. More specifically, the GTC hump gradient profile has no shearing in the center, but huge shearing in the inner and outer $1 / 4$ th of the radial domain.

These artificial features may be responsible, at least in part, for the difficulty some GK-PIC codes face in recovering flux-tube results - and gyroBohm scaling - as $\rho_{*} \rightarrow 0$. Specifically, the greater the profile shearing, the smaller the required $\rho_{*}$ required to approach the local result, as demonstrated in Ref. [1]. GYRO was designed with these considerations in mind, and uses a boundary interface algorithm which can accomodate any equilibrium profile shape: flat, peaked, or experimental. In the general case, GYRO can read the equilibrium profiles directly from an experimental dataset over the entire simulation radius.

To quantitatively demonstrate the effect of profile shear on transport scaling for this Cyclone case, we start by writing the form of the humped gradient profile as used in Refs. [3] and [7] as

$$
\frac{d T}{d r}=\left(\frac{d T}{d r}\right)_{0} \exp \left\{-[(r / a-0.5) / 0.3]^{6}\right\} \quad .
$$

While the temperature gradient above is radially-varying, the temperature itself is artificially held constant: $T(r)=T_{0}$. The maximum value of the temperature gradient is taken to match the Cyclone Base Case value of $R_{0} / L_{T, 0}=-\left(R_{0} / T_{0}\right)(d T / d r)_{0}=$ 6.9. These profiles were used by both codes in Fig. 1, and as we have already noted, introduce a region of strong profile shearing and low drive in the inner and outer 
1/4th of the radial domain. Corresponding to the four points on the GYRO curve in Fig. 1 are the radial $\chi_{i}$ profiles illustrated in Fig. 3. As an independent check on these results, the flux-tube GS2 code was run at $r / a=0.4,0.5$ and 0.6 , with the results shown as large dots in Fig. 3. The close agreement between GYRO global results and GS2 local results for this case supports the conclusion that (i) both codes are operating correctly, and (ii) local and global turbulence codes should agree for sufficiently small $\rho_{*}$. Now, if we rerun the same case, only replacing the profile of Eq. (1) with a ramped temperature gradient of the form

$$
\frac{d T}{d r}=\left(\frac{d T}{d r}\right)_{0}[1+0.3(r / a-0.5)],
$$

we observe a transition to gyroBohm scaling at a much smaller system size (larger $\left.\rho_{*}\right)$. This result is summarized in Fig. 4.

Verification of numerical convergence for gyrokinetic codes is an extraordinarily challenging issue. Because the simulation cost rises rapidly as the resolution is increased, convergence studies are rarely exhaustive. In connection with this, we have noticed that early-time estimates of $\chi_{i}$ are affected by transient phenomena and tend to overestimate the true steady-state value. For this reason, all GYRO simulations in the present paper have been run to $t \geq 900\left(a / c_{s}\right)$. The necessity of such long-time simulation is clearly illustrated in Fig. 5, where a slow relaxation of the time-averaged $\chi_{i}$ to the asymptotic level is clearly observed. In contrast, data for the largest simulation in Ref. [7] is available only up to $t=500\left(a / c_{s}\right)$. Given the behavior exhibited in Fig. 5, we believe that running the larger GTC simulations to a longer final time may result in better agreement with the local limit. Because GYRO also has the capability to simulate a flux-tube, we have plotted the result of a long-time $\left(t=1000 a / c_{s}\right)$ large-radial-box $\left(L_{r}=256 \rho_{s}\right)$ GYRO flux-tube simulation as a dotted horizontal line in Fig. 5. This is to be compared with the Dimits (dashed line) and GS2 (dot-dashed line) flux-tube results. Remarkably, all results are within about $5 \%$ of one another. Note in Fig. 5 the expanded vertical scale in comparison with Figs. 1 and 4.

In summary, we emphasize:

1. $\chi_{i}$ obtained from a global simulations are found to agree with the gyroBohm flux-tube result for sufficiently small $\rho_{*}$, as expected from basic theoretical principles;

2. the rate of approach to the gyroBohm limit with decreasing $\rho_{*}$ depends on the extent to which local growth rates exceed profile shearing rates. This has been discussed in detail in Ref. [1];

3. $\rho_{*}$-plots such as Fig. 1 are not universal, but in fact highly dependent on 
the actual profiles (as demonstrated in Fig. 4), $\mathbf{E} \times \mathbf{B}$ shear rates, as well as as on nonadiabatic electron physics and plasma shaping $[1,14]$. 


\section{Acknowledgments}

This work was supported by U.S. DOE Grants DE-FG03-95ER54309 and DE-FG0395ER54197. 


\section{References}

[1] R.E. Waltz, J. Candy, and M.N. Rosenbluth. Phys. Plasmas 9, 1938 (2002).

[2] J. Candy and R.E. Waltz. J. Comput. Phys. 186, 545 (2003).

[3] Z. Lin, S. Ethier, T.S. Hahm, and W.M. Tang. Phys. Rev. Lett. 88, 195004 (2002).

[4] F. Jenko and W. Dorland. Plasma Phys. Controlled Fusion 43, A141 (2001).

[5] F. Filbet and E. Sonnendrücker. Comput. Phys. Commun. 150, 247 (2003).

[6] A.M. Dimits, G. Bateman, M.A. Beer, B.I. Cohen, W. Dorland, G.W. Hammett, C. Kim, J.E. Kinsey, M. Kotschenreuther, A.H. Kritz, L.L. Lao, J. Mandrekas, W.M. Nevins, S.E. Parker, A.J. Redd, D.E. Shumaker, R. Sydora, and J. Weiland. Phys. Plasmas 7, 969 (2000).

[7] Z. Lin, T.S. Hahm, S. Ethier, W.W. Lee, J. Lewandowski, G. Rewoldt, W.M. Tang, W.X. Wang, L. Chen, and P.H. Diamond. Proceedings of Invited papers, 19th International Conference on Fusion Energy, Lyon, 2002 (IAEA, Vienna, 2003), TH/1-1.

[8] W. Dorland, F. Jenko, M. Kotschenreuther, and B.N. Rogers. Phys. Rev. Lett. 85,5579 (2000).

[9] S. Brunner, M. Fivaz, T.M. Tran, and J. Vaclavic. Phys. Plasmas 5, 3929 (1998).

[10] Z. Lin, T.S. Hahm, W.W. Lee, W.M. Tang, and R.B. White. Science 281, 1835 (1998).

[11] Z. Lin, T.S. Hahm, W.W. Lee, W.M. Tang, and P.H. Diamond. Phys. Rev. Lett. 83, 3645 (1999).

[12] Y. Idomura, S. Tokuda, and Y. Kishimoto. New. J. Phys. 4, 101.1 (2002).

[13] Y. Idomura, S. Tokuda, and Y. Kishimoto. Nucl. Fusion 43, 234 (2003).

[14] J. Candy and R.E. Waltz. Phys. Rev. Lett. 91, 045001-1 (2003). 


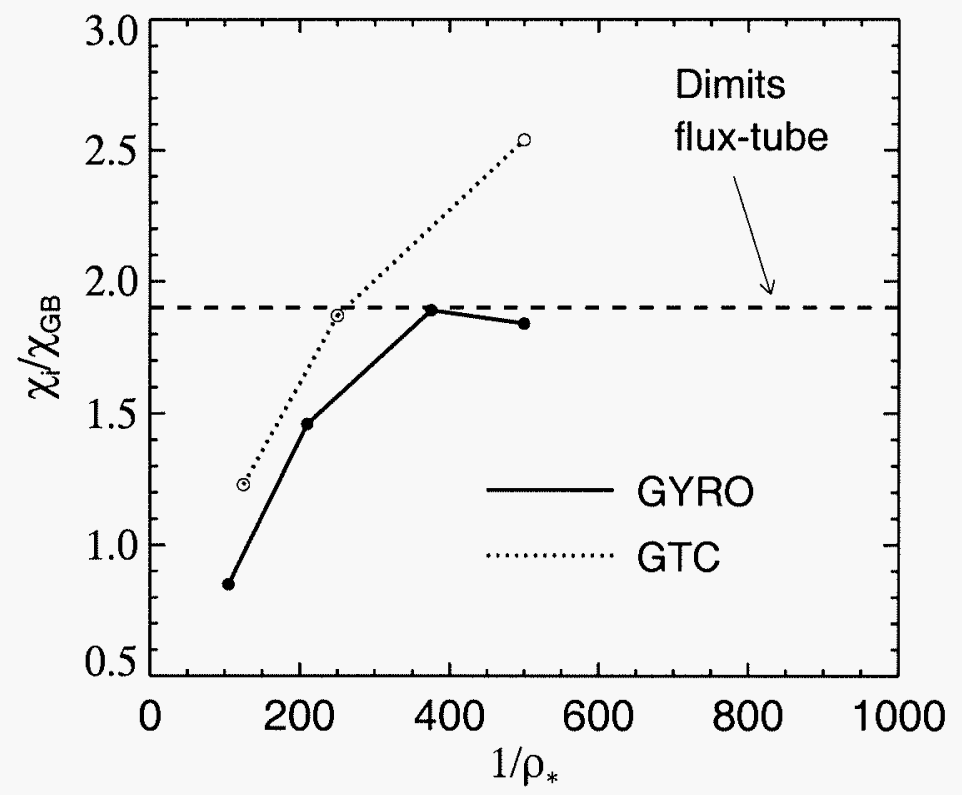

Fig. 1. Comparison of GYRO and GTC-IAEA [7] results for the global scenario originally defined in Ref. [3]. The dashed horizontal line shows the flux-tube result of Dimits [6], which is an accepted benchmark value. All GYRO points correspond to the radius $r / a=0.5$ and are averaged over the interval $400 \leq\left(c_{s} / a\right) t \leq 900$. 


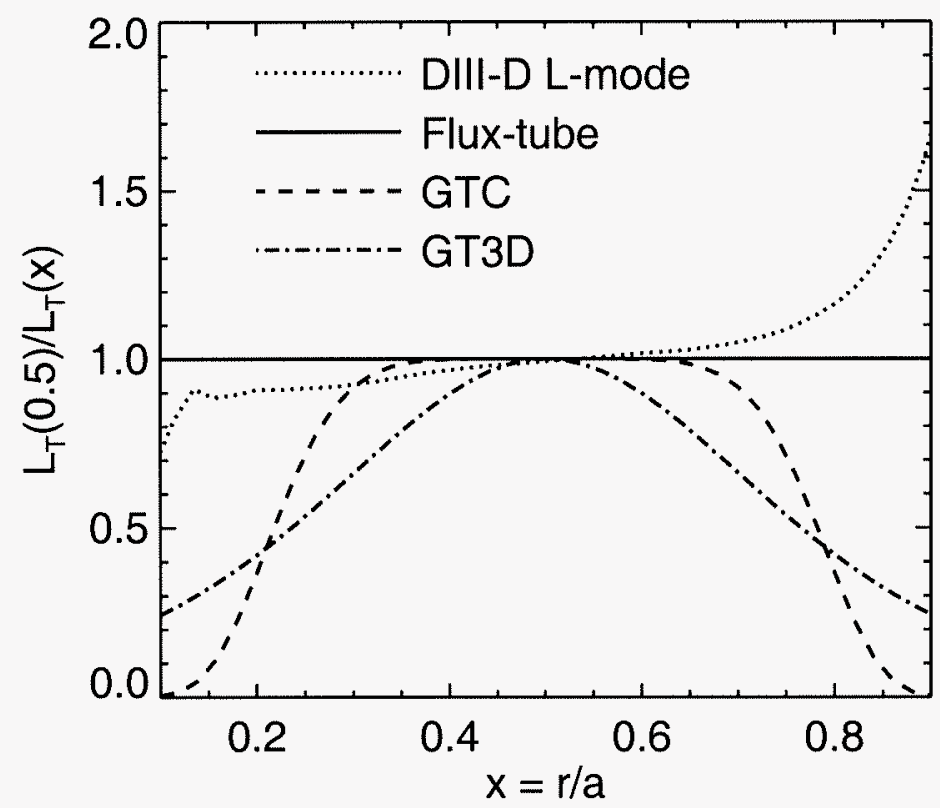

Fig. 2. Comparison of a DIII-D experimental logarithmic temperature gradient profile (dotted curve) with those assumed in various types of numerical simulations. The GT3D (dot-dashed) [12,13] and GTC (dashed) $[3,7,10,11]$ curves illustrate the peaked form routinely employed in global GK-PIC codes. The flux-tube (solid) curve shows the constant value used in flux-tube simulations. 


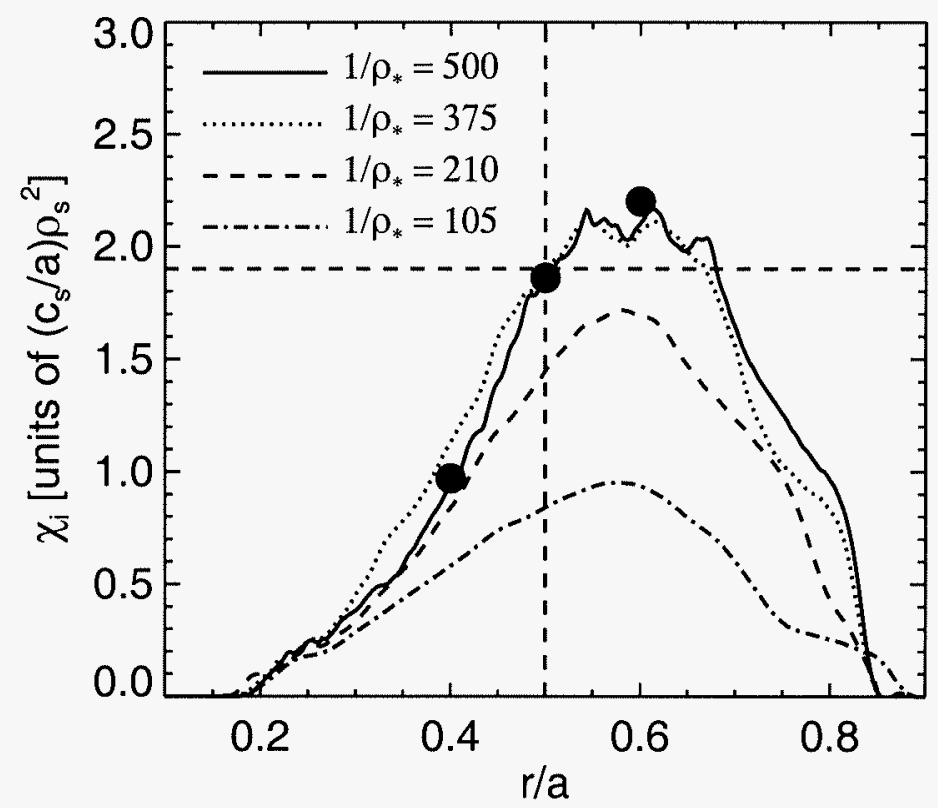

Fig. 3. Curves show radial profile of $\chi_{i}$ averaged over the time inteval $400 \leq\left(c_{s} / a\right) t \leq$ 900 , as computed by GYRO. Solid dots show results of three separate GS2 flux-tube simulations. 


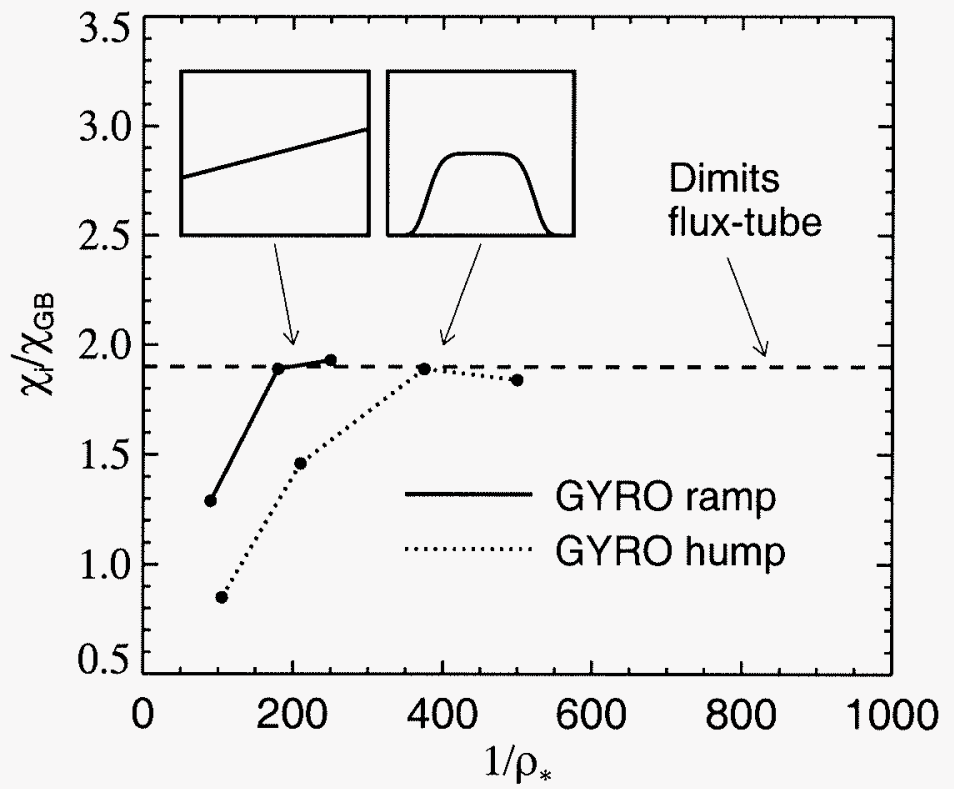

Fig. 4. Comparison of the GYRO data for the GTC hump profile (dotted curve) defined in Eq. (1), with a GYRO simulation of the ramp profile (solid curve) defined in Eq. (2). The hump results are the same as shown in Fig. 1. Note that the more realistic ramp profile exhibits gyroBohm scaling at substantially smaller system size. 


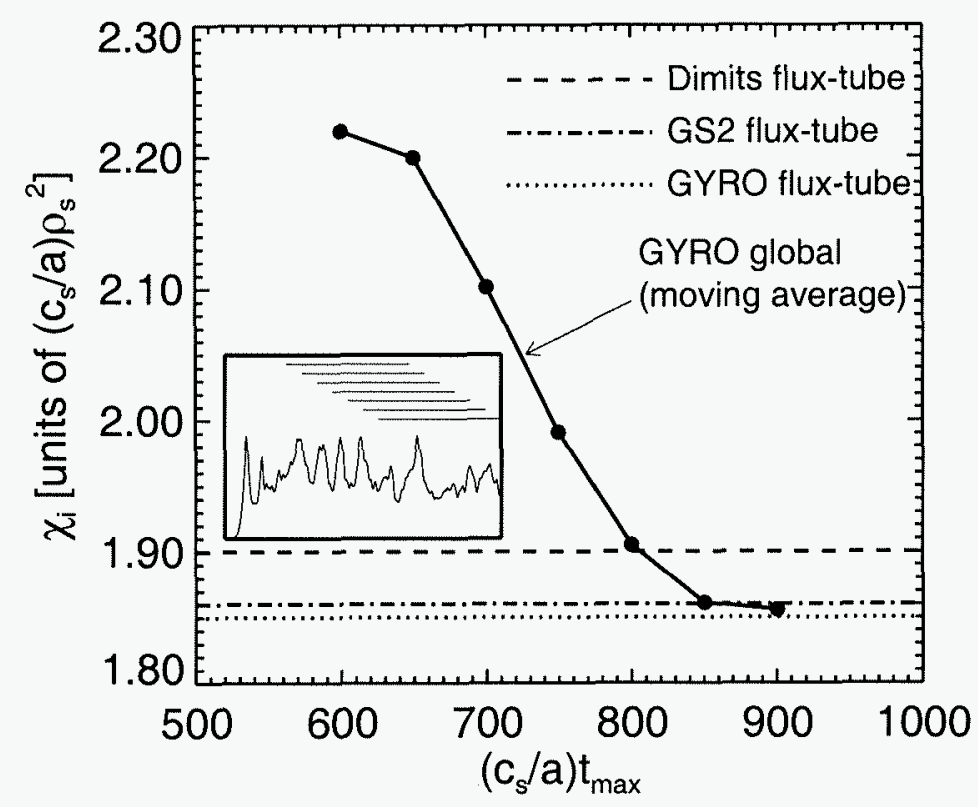

Fig. 5. Time-averaged $\chi_{i}$ from the GYRO global simulation (solid line) at $\rho_{*}=1 / 500$ for the hump scenario. The horizontal axis measures the averaging interval: a point at $t_{\max }$ indicates an average over $\left(c_{s} / a\right) t_{\max }-400 \leq\left(c_{s} / a\right) t \leq\left(c_{s} / a\right) t_{\max }$. Also, three horizontal lines show the final result from flux-tube calculations: Dimits [6] (dashed), GS2 (dot-dash) and GYRO (dotted). The GYRO flux-tube result was averaged over $500 \leq\left(c_{s} / a\right) t \leq 900$. Plot-in-plot shows the instantaneous $\chi_{i}$ over $0 \leq\left(c_{s} / a\right) t \leq 900$, including horizontal bars which denote averaging windows. 